\title{
Stacked Janus Device Concepts: Abrupt pn-Junctions and Cross-Plane Channels
}

Palsgaard, Mattias Lau Nøhr; Gunst, Tue; Markussen, Troels; Thygesen, Kristian Sommer; Brandbyge, Mads

\section{Published in:}

Nano Letters

Link to article, DOI:

10.1021/acs.nanolett.8b03474

Publication date:

2018

Document Version

Peer reviewed version

Link back to DTU Orbit

Citation (APA):

Palsgaard, M. L. N., Gunst, T., Markussen, T., Thygesen, K. S., \& Brandbyge, M. (2018). Stacked Janus Device Concepts: Abrupt pn-Junctions and Cross-Plane Channels. Nano Letters, 18(11), 7275-7281.

https://doi.org/10.1021/acs.nanolett.8b03474

\section{General rights}

Copyright and moral rights for the publications made accessible in the public portal are retained by the authors and/or other copyright owners and it is a condition of accessing publications that users recognise and abide by the legal requirements associated with these rights.

- Users may download and print one copy of any publication from the public portal for the purpose of private study or research.

- You may not further distribute the material or use it for any profit-making activity or commercial gain

- You may freely distribute the URL identifying the publication in the public portal

If you believe that this document breaches copyright please contact us providing details, and we will remove access to the work immediately and investigate your claim 


\title{
Stacked Janus device concepts: abrupt $p n$-junctions and cross-plane channels
}

\author{
Mattias Palsgaard, ${ }^{*, \dagger, \uparrow}$ Tue Gunst, ${ }^{*,+, \uparrow}$ Troels Markussen, ${ }^{\dagger}$ Kristian Sommer \\ Thygesen,,$₫$ and Mads Brandbyge \\ †Synopsys-QuantumWise, Fruebjergvej 3, Postbox 4, DK-2100 Copenhagen, Denmark \\ $\ddagger$ Department of Micro- and Nanotechnology (DTU Nanotech), Technical University of \\ Denmark, DK-2800 Kgs. Lyngby, Denmark \\ ICenter for Nanostructured Graphene (CNG) \\ $\S$ Department of Physics, Center for Atomic-scale Materials Design, Technical University of \\ Denmark, DK-2800 Kgs. Lyngby, Denmark \\ E-mail: mlnp@nanotech.dtu.dk; tue.gunst@nanotech.dtu.dk
}

\begin{abstract}
Janus transition metal dichalcogenides with a built-in structural cross-plane (cp) asymmetry have recently emerged as a new class of $2 \mathrm{D}$ materials with a large cp dipole. Using first principles calculations, and a tailored transport method, we demonstrate that stacking graphene and MoSSe Janus structures result in record high homogeneous doping of graphene and abrupt, atomic-thin, cross-plane $p n$-junctions. We show how graphene, in contrast to metals, can act as electrodes to Janus stacks without screening the cp dipole, and predict a large photo-current response dominated by a cp transport channel in a few-layer stacked device. The photo-current is above that of a corresponding thin-film silicon device illustrating the great potential of Janus stacks f.ex. in photovoltaic devices.
\end{abstract}


Controlling the built-in or environmental planar symmetry of two-dimensional materials paves the way for the next level of rich 2D physics phenomena. One promising example is the emerging Janus transition metal dichalcogenides (TMD's), such as MoSSe, where a strong planar asymmetry, due to different atomic species on each side, generates a large dipole across the plane. ${ }^{1,2}$ Other recent relevant examples include how the stacking environment of $2 \mathrm{D}$ materials can be used to tune properties like the optical gap,,${ }^{3,4}$ mobility, ${ }^{5}$ interlayer excitons ${ }^{6,7}$ and local doping. ${ }^{8,9}$ One key focus has so far been to find TMD's with different band gaps. ${ }^{10}$ However, the properties of Janus MoSSe are far more interesting than simply an expected band gap in between the two out-of-plane symmetric parents $\left(\mathrm{MoS}_{2}\right.$ and $\left.\mathrm{MoSe}_{2}\right)$.

While the structural stability ${ }^{11}$ and the potential usefulness of MoSSe for solar water splitting ${ }^{12-14}$ and band gap engineering ${ }^{15}$ have been examined previously, device properties and the interaction of the Janus dipole with other stacking materials and electrodes have not been discussed so far. Here we show how stacking graphene and Janus structures gives a route to record high homogeneous doping of graphene in abrupt $p n$-junctions. In addition, semi-metallic leads such as graphene, are needed to avoid the dipole cancellation due to the screening that occurs with metal contacts. Such effects are crucial to address for the potential application of Janus structures in devices. In addition, we provide for the first time estimates of the photo-current generated in device configurations. Harvesting solar energy through photovoltaics (PV) represents a promising technology as a replacement for burning fossil fuels and the potential of Janus TMD's as a new alternative or constituent in PV devices in the future is discussed. We calculate the ballistic single-particle charge transfer with a transport method tailored to stacked Janus and Janus-graphene devices, neglecting excitonic effects. Computational details are described in the Methods section.

Monolayer Janus MoSSe. The origin of the out-of-plane asymmetry and the resulting Janus dipole mechanism is illustrated in Fig. 1 for a monolayer MoSSe system. A layer of Mo is sandwiched in between a layer of $\mathrm{S}$ and a layer of Se atoms. From the calculated bandstructure in Fig. 1(a) we find a direct band gap, located at the $K$ and $K^{\prime}$-points, with a 

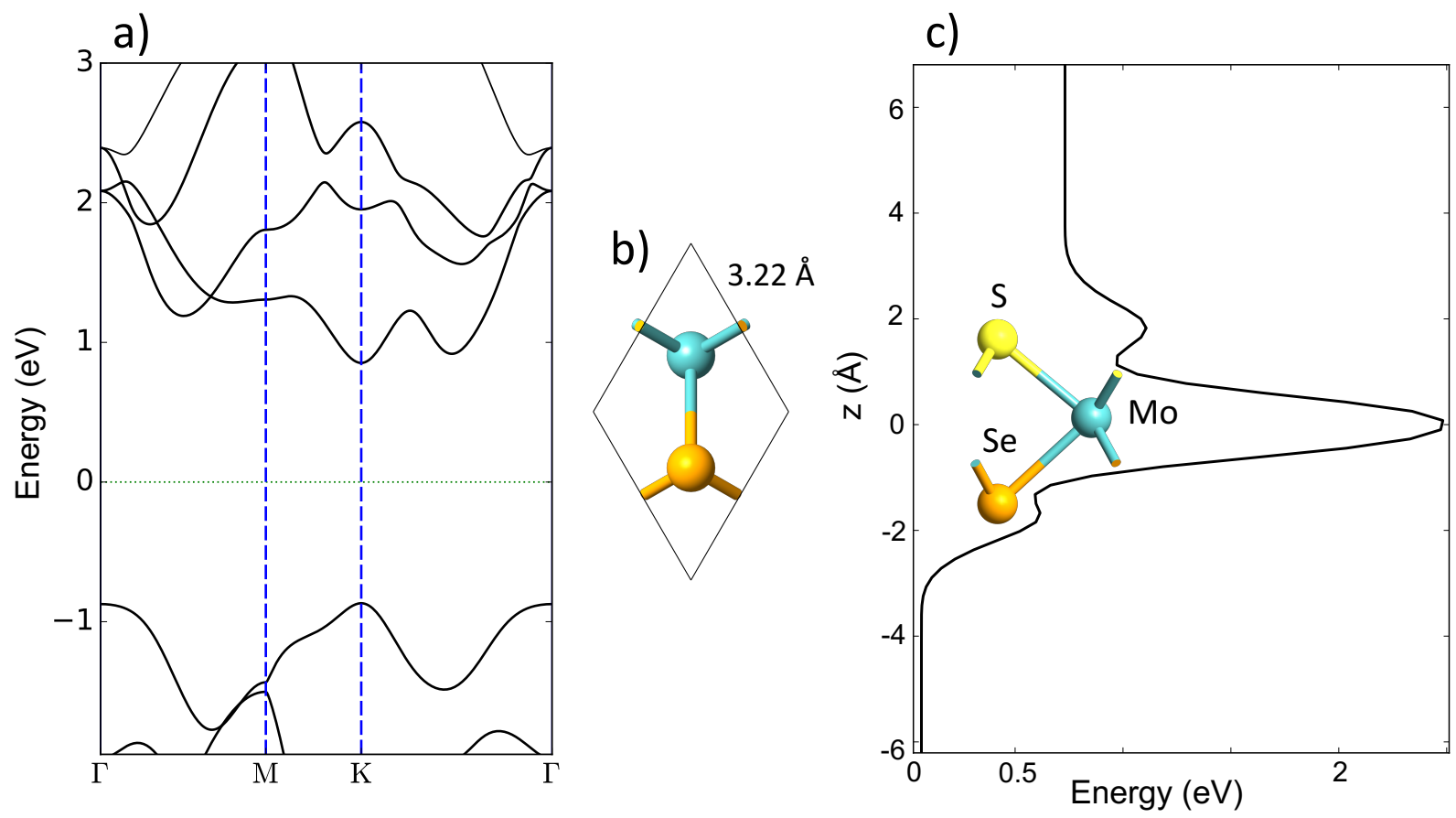

Figure 1: Janus dipole mechanism. a) Bandstructure and b) Top view of the unit cell of monolayer Janus MoSSe. c) Electrostatic difference potential averaged in the plane of monolayer MoSSe plotted as a function of the cross-plane (cp) position, $z$.

value of $1.72 \mathrm{eV}$ which corresponds nicely to the $1.77 \mathrm{eV}$ optical band gap previously reported for Janus MoSSe. ${ }^{1,14,16}$

In Fig.1(c) we show the electrostatic difference potential averaged over the xy-plane as a function of the z-position. This clearly illustrates the built-in cp dipole field with a potential difference of $0.66 \mathrm{eV}$ between the two sides. Previous studies discuss the structural asymmetry and a large out-of-plane piezoelectric polarization of Janus materials. ${ }^{1,17}$ A simple explanation of the cp dipole field, observed in Fig. 1(c), can be found in the fact that $\mathrm{S}$ and Se atoms have slightly different electronegativity, which for a full layer gives a large difference in work-function between the two surfaces and induces a dipole across the slab. We employ Neumann boundary conditions at the vacuum boundaries when solving the Poisson equation $^{5,18}$ allowing for self-consistent calculations of the surface dipole field.

Stacked Janus MoSSe. By stacking multiple Janus MoSSe layers on top of each other it is possible to stack the dipoles of the individual layers and obtain an atomically thin pn- 
junction across the multilayer system. We demonstrate this by projecting the bands on each layer in a trilayer Janus MoSSe system in Fig. 2(a). The bands are colored according to the

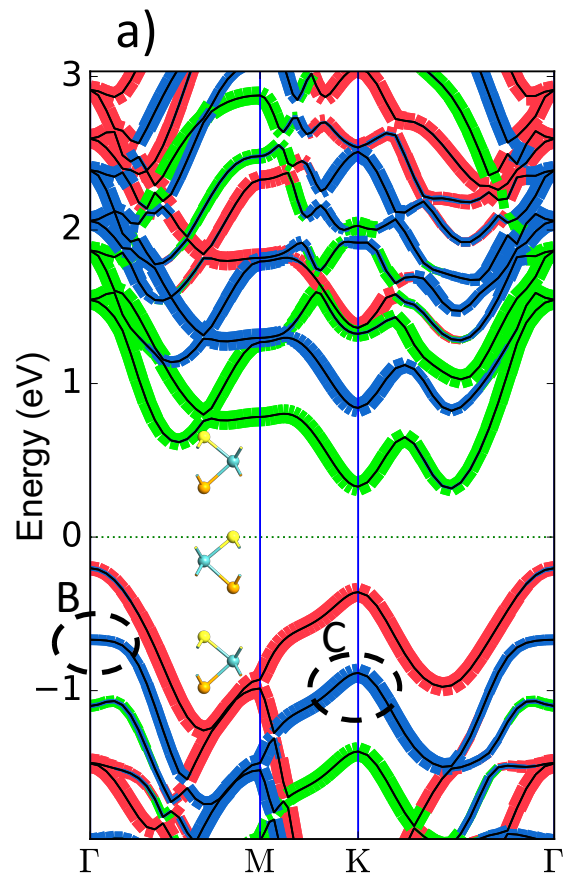

b)
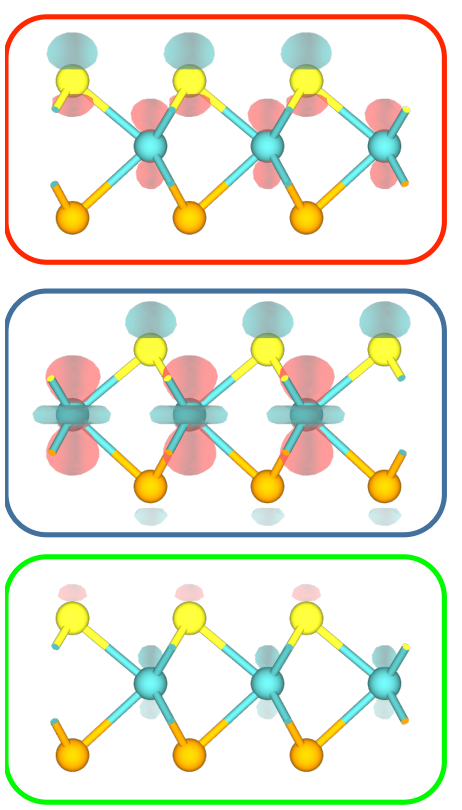

c)

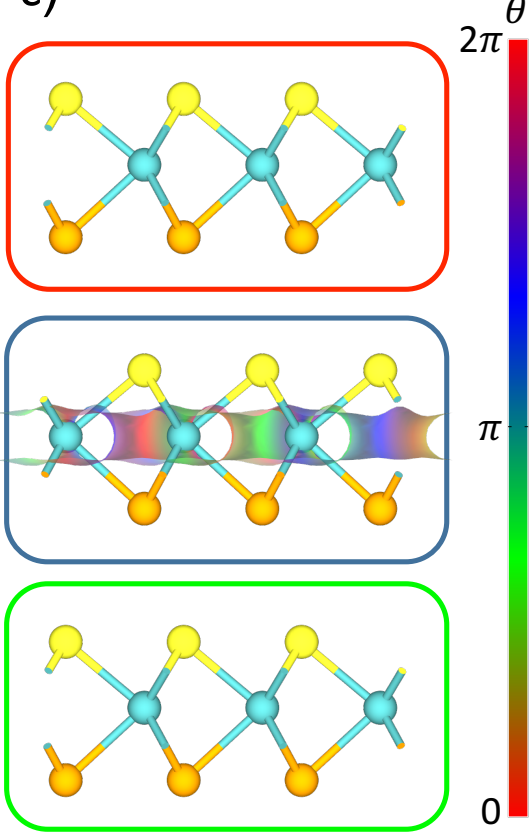

Figure 2: a) Bandstructure of trilayer Janus MoSSe projected onto the seperate layers. The structure is shown in the inset. Line thickness and the color red, blue and green lines represents projections on the top, middle and bottom layers, respectively. b) Cross-plane channel: Bloch state isosurface of the $\Gamma$-point state highlighted by point B in a). c) In-plane channel: Bloch state isosurface of the $K$-point state highlighted by point $\mathrm{C}$ in a). The color shows the phase of the wavefunction.

projection on each of the three layers, with the bottom layer having a Se surface and the top layer has a S surface. Clearly, the top (red) layer with the S-terminated surface is more $p$-like, the bottom (green) layer with the Se-terminated surface is $n$-like and added layers each obtain bands shifted by $\sim 0.5 \mathrm{eV}$. This trend applies for 1-3 layers until the 4th layer is added, where the conduction band minimum and valence band maximum intersect the Fermi level and a $p n$-junction is obtained. Adding additional layers to the stack does not increase the dipole due to charge transfer between the top and bottom layers. In addition, we find that stacked Janus MoSSe gets an indirect band gap as the valence band maximum shifts from the $K, K^{\prime}$-points to the $\Gamma$-point. However, rather than discussing this in terms 
of a decreasing band gap, we focus on the fact here that stacking Janus layers is a way of obtaining atomically thin pn-junctions inherently through the Janus dipole effect. This is interesting for the potential applications in e.g. photodiodes, which we will return to after a discussion about how to contact such a Janus surface induced dipole $p n$-junction and identifying possibly photo-current active channels.

Photo-current active channels, i.e. states that facilitates charge transfer through the $p n$ junction, will have a high degree of spatial distribution across the different layers. This is not the case for the highly-dispersive valence bands near the $K$-valley (marked C in Fig. 2(a)). Looking at the valence band in Fig. 2(a) near the $\Gamma$-point (marked B), we find a state that has a low in-plane dispersion but is delocalized in the cp-direction (the blue line projected band is thinner at the $\Gamma$-point as compared to the $K$-point due to contributions from all three layers). We highlight this through Fig. 2(b) showing the isosurface of the Bloch state located near the $\Gamma$-point that illustrates the spread across the slab of this state. In Fig. 2(c) we show the corresponding valence band state at the $K$-point (also highlighted in 2(a)) with the same isovalue. In contrast to the $\Gamma$-point state, the $K$-point state is an in-plane channel; it is highly localized in the middle MoSSe monolayer showing no delocalization in the cp-direction.

While the valence states in the $K$-valley are merely a superposition of states localized in each layer, illustrated in Fig. 2(c), this is not the case for the $\Gamma$-valleys - a point so far overlooked in the literature. The three states originating from different layers at the $\Gamma$-point are dissimilar and the one originating from the middle MoSSe layer is the most spread out. We conclude that the state shown in Fig. 2(b) is a cp-channel distributed across all layers of the system. Due to the low in-plane dispersion it exhibits a molecular orbital-like character. Contrary to a usual in-plane transport mechanism such low-dispersive bands can therefore be a good indication of cp-transport activity. This cp-channel will be responsible for the large photo-current response, as shown below.

Also notable is the difference in effective masses (inverse curvature) of the valence bands 
around the $\Gamma$-point. From Fig. 2(a) we see that the effective mass of the valence bands around the $\Gamma$-point increases significantly for the second layer and changes sign for the third layer. This change is caused by the cp-field between the layers and can be reversed by creating an external field in the opposite direction of the built-in dipole using e.g. gates of opposite polarity above and below the structure. We therefore make a conjecture that the photo-current active band will be highly tunable by external gating. We will explicitly show that is this indeed the case when discussing the device simulations.

Stacked Janus MoSSe and graphene. Next we discuss the concept of Janus dipole doping of graphene and show how graphene can be used as electrodes for Janus MoSSe devices. Stacked systems are illustrated in Fig. 3(a,b). The stacking of dipoles, shown in the multilayer MoSSe structures, presents a unique opportunity for creating atomically thin pn-junctions in the cp-direction for harvesting of solar energy. To facilitate such a device, connections to metallic leads are necessary. However, simply adding bulk metal leads to the top and bottom layers would completely screen the built-in dipole ruining the $p n$-junction as shown in the Supporting Information (Fig. S1-S4). This screening could turn out to be a major challenge for exploiting the built-in cp-dipole and the unique properties of Janus materials. We expect semi-metallic 2D materials to screen the cp-field less effectively and have therefore examined the effect of adding graphene monolayers to the top and bottom of multilayer MoSSe.

In Fig. 3(c) we show the projected bandstructure of three layers of MoSSe with graphene added to the top and bottom of the structure as shown in Fig. 3(b). A hexagonal supercell of graphene on top of MoSSe (shown in Fig. 3(a)) was obtained with four and seven repetitions of the MoSSe and graphene unit cells, respectively. A compressive strain of $\sim 1 \%$ is added to graphene in this supercell. We chose to strain only graphene since we are interested in the details of the MoSSe electronic structure while graphene is simply used as a lead. ${ }^{19-21}$ Importantly, we see from Fig. 3(c) that while some screening of the cp-dipole occurs, it mostly survives. As indicated in the bandstructure the difference in energy between the 


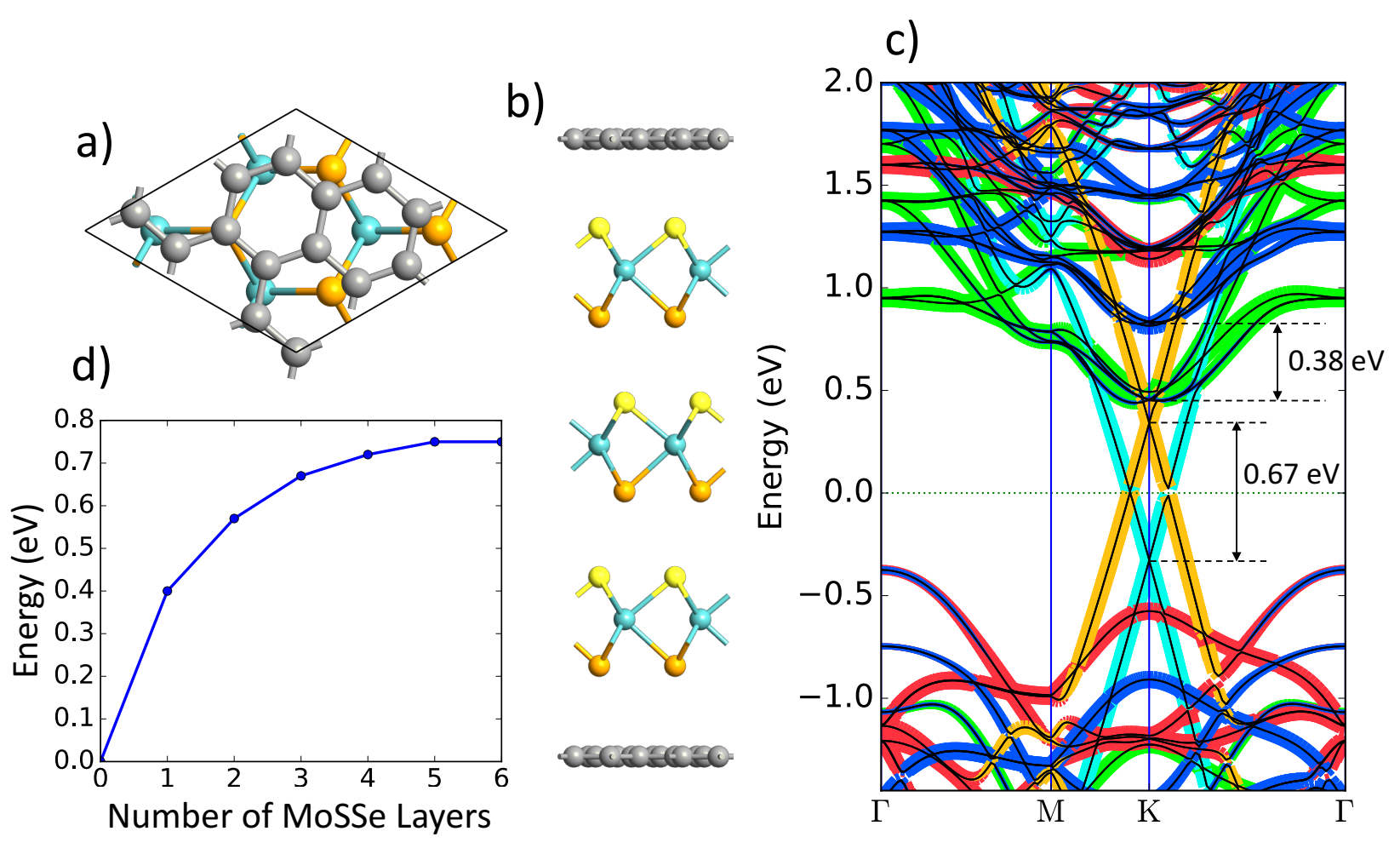

Figure 3: a) Top view of 2D hexagonal supercell of graphene-MoSSe stack. b) Side view of trilayer MoSSe sandwiched between graphene. c) Projected bandstructure of trilayer Janus MoSSe with graphene added on top and at the bottom. Red, blue and green lines represents projections on to the first, second and third MoSSe layer, respectively. d) Energy-shift between the two graphene Dirac-points for varying number of Janus layers.

states localized on adjacent MoSSe layers is reduced from $0.51 \mathrm{eV}$ to $0.38 \mathrm{eV}$ as a result of screening effects when adding graphene on either side. Graphene is therefore promising as electronic leads to Janus MoSSe preserving the dipole effect.

Interestingly the graphene layers are strongly doped $n$ - and $p$-type on the top and bottom respectively. In fact, the charge neutrality points in the graphene sheets are separated by $0.67 \mathrm{eV}$ by the intense field created by the three layer MoSSe structure. The concept of Janus dipole doping is summarized is Fig. 3(d). The doping effect increases up to a $0.8 \mathrm{eV}$ separation of the charge neutrality points for six layers of MoSSe which is a very high homogeneous doping of graphene. This effect could be used to effectively dope graphene in a non-intrusive way where no dopant atoms need to be implanted in the pristine graphene sheet and the doping can be controlled by the number of stacked MoSSe layers used. The maximal Fermi- 
level shift of $0.8 \mathrm{eV}$ in Fig. 3(d) corresponds to a carrier density of $1 \times 10^{13} \mathrm{~cm}^{-2}$. This falls in between typical doping values of a few times $10^{12} \mathrm{~cm}^{-2}$, e.g. achieved with molecular doping ${ }^{22}$ or backgates, ${ }^{23}$ and the ultrahigh carrier densities of $\sim 10^{14} \mathrm{~cm}^{-2}$ obtained by electrolytic gating. ${ }^{24,25}$ Stacked Janus-graphene structures hereby give a promising route to abrupt $p n$ junctions, something that has previously required complex double-gated configurations, ${ }^{26}$ with a homogeneity exceeding that of electrolytic or ionic gating and with the outer layers (graphene or Janus) being accessible due to the internal Janus-doping. Next we will discuss the potential optoelectronic applications of Janus materials and the transport model used.

Transport model of stacked devices. In Fig. 4(a) we illustrate the envisioned device consisting of MoSSe sandwiched between two graphene electrodes. Even for a monolayer of Janus MoSSe we see in Fig. 4(b) that a remarkable $0.4 \mathrm{eV}$ shift in energy occurs between the graphene sheets which are separated by less than $1 \mathrm{~nm}$ while the MoSSe bandstructure remains intact. In order to check that a current can be injected from the doped graphene layers through Janus MoSSe, we calculate the cp-transmission from the Landauer-Büttiker expression

$$
T_{c p}=\operatorname{Tr}\left\{\Gamma_{L B} G \Gamma_{R T} G^{\dagger}\right\}
$$

where $G$ is the retarded Green's function of the device and $\Gamma_{L B / R T}$ is the broadening coming from the left bottom and right top graphene leads respectively, as shown in Fig. 4(a). To obtain $\Gamma_{L B / R T}$ the full $\Gamma_{L}$ and $\Gamma_{R}$ matrices for the leads indicated in Fig. 4(a) were calculated recursively using the DFT-NEGF code provided by the QuantumATK software. ${ }^{28}$ Subsequently $\Gamma_{L / R}$ was projected onto $\Gamma_{L B / R T}$ parts by removing indices of the full matrices related to the other layers (Supporting Information, Fig. S5). In this way cp-transport is considered with the transport direction still inside the plane. Fig. $4(\mathrm{c})$ shows $T_{c p}(E)$ for the monolayer MoSSe system with monolayer graphene leads on either side. Clearly transmission channels are available and a high transmission is seen for a bias window above the MoSSe 

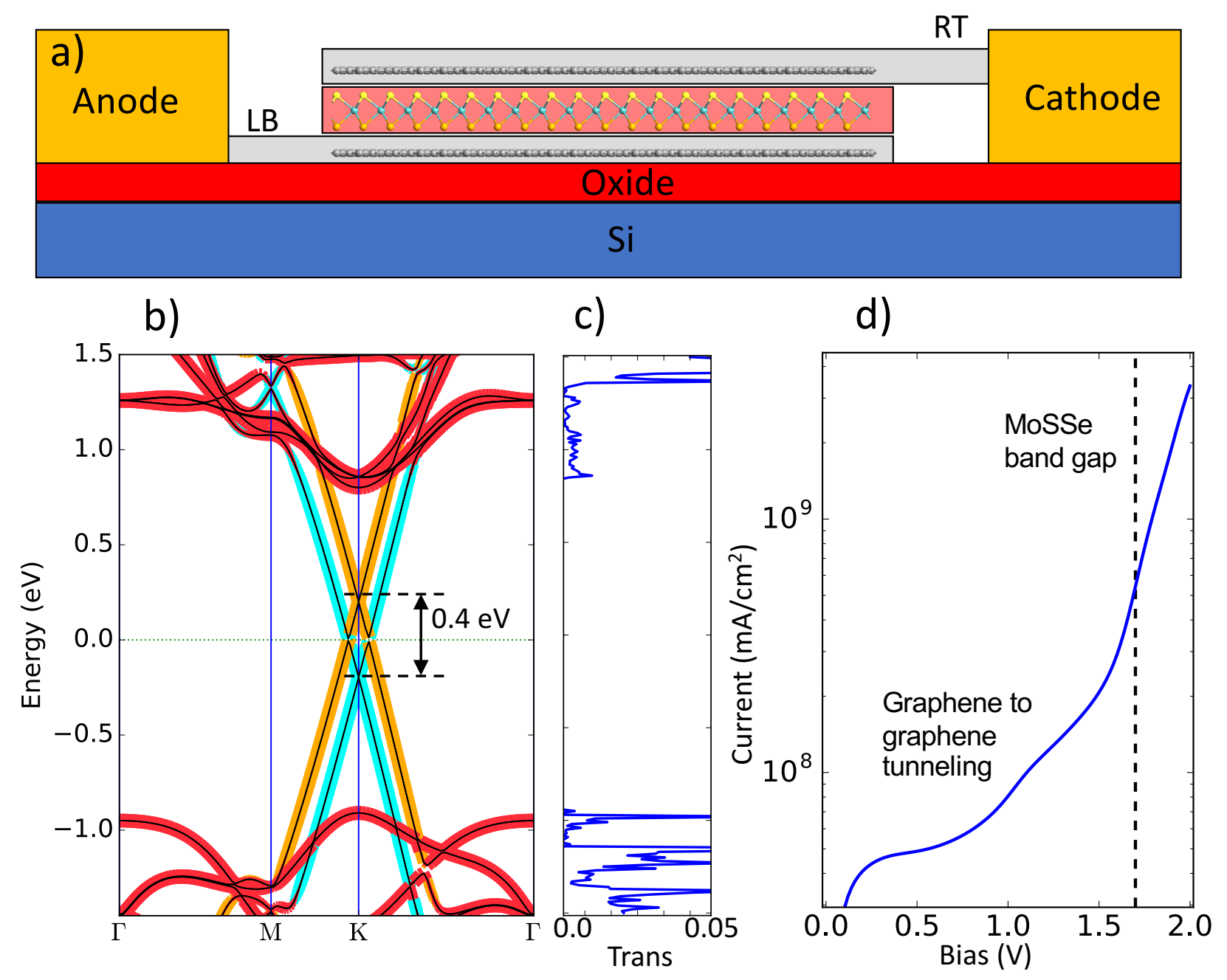

Figure 4: a) Envisioned device with graphene and Janus stacks to avoid dipole cancellation and achieve an abrupt $p n$-junction. b) Projected bandstructure of monolayer Janus MoSSe with graphene on top and at the bottom. Red line represents projection on the MoSSe, yellow and cyan lines projection onto the top and bottom graphene layer, respectively. c) Electron transmission from the left bottom graphene electrode to the right top graphene electrode. d) IV-characteristics ${ }^{27}$ of the graphene-Janus stacked device at $300 \mathrm{~K}$. The current is evaluated from the zero bias transmission function.

gap where states are available in all three monolayers. A very small transmission directly from one graphene layer to the other is also seen at the Fermi level, where the graphene cones intersect. We consequently find a low bias regime with a direct graphene-graphene tunneling current and a high bias regime with bulk Janus transport in the IV-characteristics shown in Fig. 4(d).

Janus MoSSe device photo-current. Next we consider the electron transmission and 
photogenerated current for a three layer MoSSe device, Fig. 5(a). Since we have shown how

a)
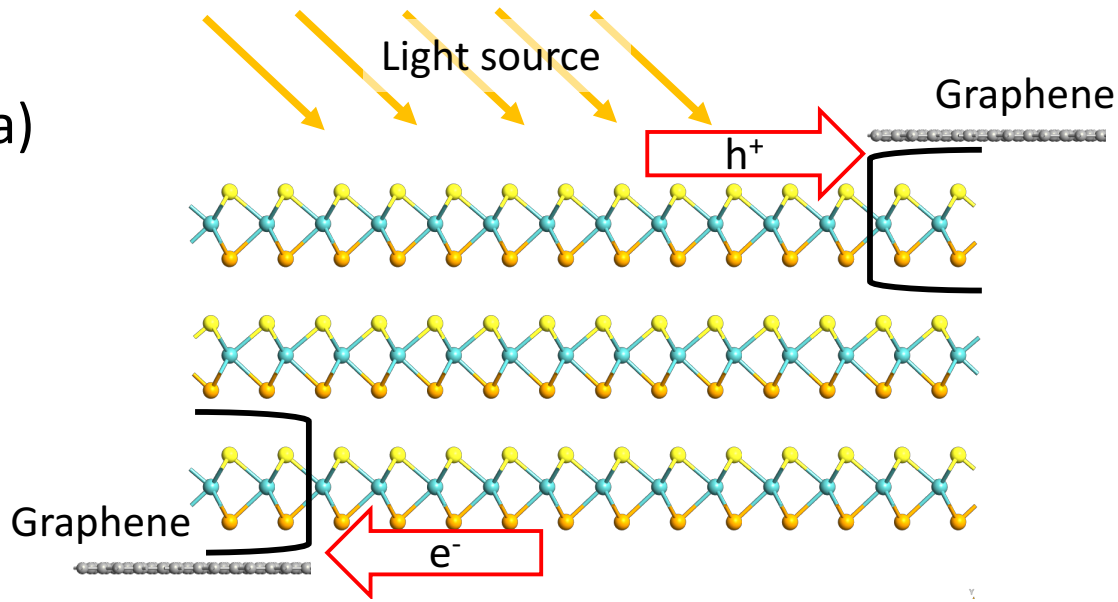

b)

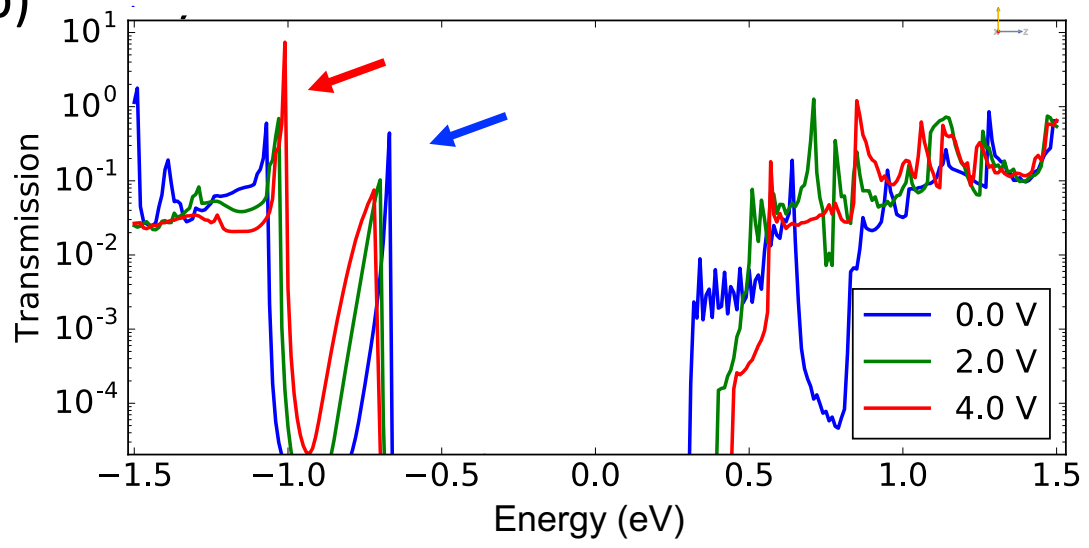

Figure 5: a) Trilayer Janus MoSSe device structure used to calculate transmission from the top to the bottom layer. b) Out-of-plane transmission for different values of the gate voltage illustrating the tunability of the cp-channel.

screening of the cp-dipole can be avoided by semi-metallic graphene leads, we imagine a device where MoSSe is directly exposed to light and the connection to graphene is made further away from photo-active area (or is considered transparent as compared to the full stack). In this case, we can consider transport directly from the top left to the bottom right MoSSe layer, as illustrated in Fig. 5(a), which makes the calculations computationally feasible. In order to examine the tunability of the photo-responsive cp-channel we included two metallic gates separated by $40 \AA$ above and below the three layer structure. A voltage of positive/negative polarity was applied to the top/bottom gate to control a field in the 
opposite direction of the built-in dipole field of the MoSSe. In this device, the dipole field creates a $p n$-junction in the cp-direction that is capable of separating holes and electrons into the top and bottom layers, hereby generating a photocurrent between the layers. The physical transport mechanism is interlayer tunneling via the cp-channel discussed previously.

The cp Landauer-Büttiker transmission (1) is shown in Fig. 5(b) for three different values of the gate voltage. First we see a clear peak in the transmission at $-0.67 \mathrm{eV}$ for the system without applied gate voltage (indicated by a blue arrow) which is due to the cp-channel. The delocalization of the cp-channel across the MoSSe layer enables it to carry the cpcurrent effectively. Furthermore, the almost dispersion-less bands of the cp-channel, shown in Fig. 2(a), leads to a sharply peaked van Hove-like transmission. It is possible to tune the dispersion of the band by applying the gate voltage to creates an electrical field opposite to the built-in field. We observe how the peak is broadened due to the increased band dispersion and resulting decrease in density of states for the cp-channel. Furthermore, as the gate voltage is increased another sharp transmission peak emerges at lower energy (indicated by a red arrow). This peak is related to the $\Gamma$-point valence state, shown in Fig. 2(a). The increased gate voltage tunes the effective mass of this band making it nearly dispersionless in the plane, and create a new cp-channel (red arrow) replacing the previous one (blue arrow). This shows how it is possible to tune the in-plane effective mass and the cp-transport properties substantially with a gate voltage.

In Fig. 6(a) we plot the photo-excited cp-current for a two and three layer Janus-MoSSe device compared to that calculated for a $20 \mathrm{~nm}$ thick silicon $p n$-junction. ${ }^{30,31}$ Interestingly, the extremely thin (0.5-1 nm) Janus photo-devices generates a current above that of the 20-40 times thicker silicon device considered, which underlines the great potential of Janus materials in thin-film photodiodes. Comparing the two and three layer MoSSe photo-currents, we see that the generated photo-current is almost independent of the thickness: the absorption of photons is improved by adding an additional Janus-MoSSe layer while the tunneling transmission is reduced at the larger distance from the top to the bottom layer. These 

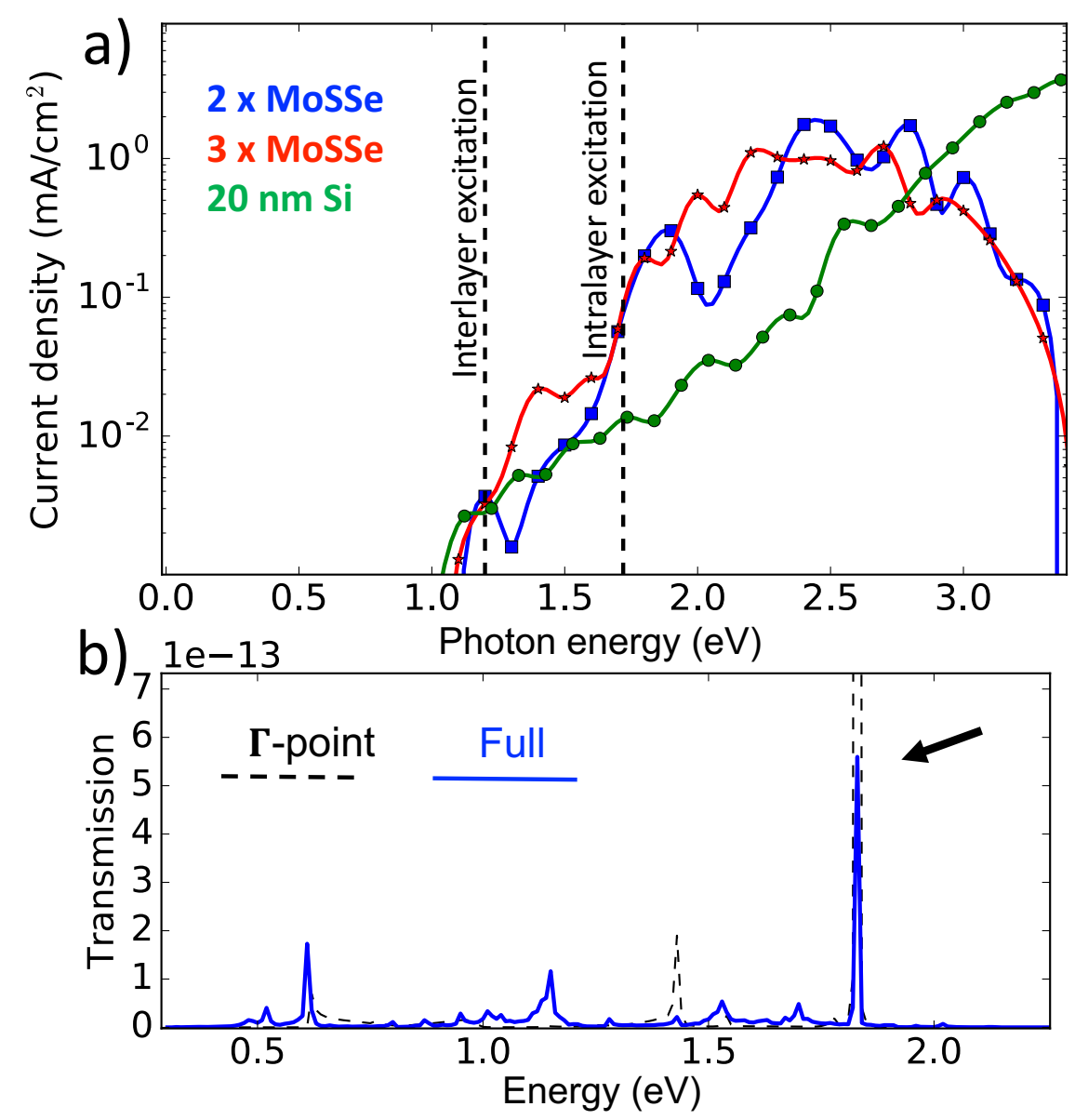

Figure 6: a) Photocurrent density ${ }^{27}$ as a function of photon energy for the three layer structure shown in Fig. 5(a). The current was at each energy weighted by the flux of the solar AM1.5 reference spectrum. ${ }^{29}$ b) Photon mediated transmission as a function of energy for the $2.5 \mathrm{eV}$ photon. The full k-averaged transmission shows a dominant peak at $1.83 \mathrm{eV}$ which is traced back to the cp-channel in a region around the $\Gamma$-point as illustrated by the $\Gamma$-point transmission.

two mechanisms almost cancel each other, and we find that there is only a small difference between the photo-excited cp-current for two and three layers of Janus-MoSSe.

We notice that for both Janus-devices there is a significant contribution to the current from photons with energies below the $1.72 \mathrm{eV}$ gap of monolayer Janus-MoSSe (as indicated by the vertical dashed lines in Fig. 6(a)). This current originates from interlayer excitations where the conduction band and valence band are in different Janus layers, effectively reducing the transport gap of the Janus-MoSSe device.

The origin of the large photo-current is illustrated in Fig. 6(b), showing the photon- 
mediated transmission for photons with an energy of $\hbar \omega=2.5 \mathrm{eV}$. The highest transmission (indicated by an arrow in Fig. 6(b)) is located at an energy of $1.83 \mathrm{eV}$, which is the final state energy of an excitation starting from the $\Gamma$-point cp-channel shown in Fig. 2(b) (further highlighted by the $\Gamma$-point transmission, which illustrates that the dominant peak can be traced back to a region in the vicinity of the $\Gamma$-point). This clearly shows that the $\mathrm{cp}$ photocurrent in multilayer Janus-MoSSe is dominated by the cp-channel. Furthermore, we notice that at photon energies above $2.5 \mathrm{eV}$ the photo-current starts to fall off. While this high energy region is less relevant in the context of solar cell applications it is still interesting to explain this somewhat unusual behavior. Starting at the cp-channel energy, point B in Fig. 2(a) we see that for energies photon above $2.5 \mathrm{eV}$ one enters a regime where there are no bands in the opposite layer. Hereby, the cp-current is reduced and eventually the current starts to run in the opposite direction. This behavior is only captured by transport calculations which can account for the current direction of the generated electrons.

Finally, we point out the potential use of 2D Janus TMD's in tandem structures. Combining for instance a few MoSSe Janus layers with silicon thin-films could increase the broad spectrum absorption of light, especially in the low energy regime where silicon absorbs weakly. We chose in this study to focus on MoSSe due to its recent experimental realization. However, with recent efforts to create databases of emerging $2 \mathrm{D}$ materials ${ }^{10}$ it is possible to identify other Janus candidates that have a lower band gap and a similar expected dipole effect. For instance we notice that CrSSe and ZrSSe have band gaps of approximately $0.5 \mathrm{eV}$ and $0.8 \mathrm{eV}$ respectively, and that certain bulk materials with a large dipole effect like BiTeI should be exfoliable. This underlines some of the prospects in studying new Janus materials to increase device performance and how Janus materials could play an important role as alternatives or constituents in PV devices in the future.

Conclusion. In summary, we have employed first principles calculations and a tailored transport method to demonstrate three new concepts rooted in the built-in structural outof-plane asymmetry of novel 2D Janus materials: The Janus dipole doping effect, the Janus- 
graphene electrode stacks for avoiding dipole cancellation, and the Janus cross-plane channels dominating the photo-current response. Janus dipole doping allows one to achieve so far unprecedented homogeneous doping levels of graphene in abrupt, atomic-thin, $p n$-junctions. In particular, the graphene leads are crucial for device applications of Janus materials due to the dipole cancellation with conventional metallic electrodes. Among many possible applications of Janus stacks we focused on the photo-voltaic properties, and showed how Janus cross-plane channels give rise to a photo-current exceeding that of thin-film silicon devices.

\section{Methods}

All results presented in this paper are obtained using the QuantumATK software. ${ }^{28}$ Electronic structure calculations are calculated using Density Functional Theory (DFT) with a double zeta polarized LCAO basis set and a $k$-point density corresponding to (51x51)sampling in the minimal unitcell. Local Density Approximation (LDA) is used for the exchange-correlation potential. In the out-of-plane direction over $30 \AA$ of vacuum is included and Neumann conditions are employed at the boundary. Transport calculations are performed with the usual DFT-NEGF approach ${ }^{32}$ using $301 \mathrm{k}$-points in the transverse direction. We chose a lattice parameter $(3.22 \AA)$ and interlayer distance $(3.14 \AA)$ as calculated previously. ${ }^{14}$ Atomic coordinates were relaxed until all interatomic forces were below $0.02 \mathrm{eV} / \AA$. Guan et al. ${ }^{14}$ found $A^{\prime} B$ stacking order to have the lowest binding energy for bilayer Janus MoSSe unlike the conventional TMD's that all exhibit $A A^{\prime}$ stacking. However since the difference in binding energy between $A A^{\prime}$ and $A^{\prime} B$ is only $2 \mathrm{meV}$ both configurations are likely to be found in experiments. We therefore choose to study $A A^{\prime}$ stacking which allows for straightforward comparison with previous studies of devices using other TMD's.

As derived in previous studies ${ }^{30,33-35}$ the first order correction to the current into lead 
$\alpha=L B / R T$ due to absorption of $N$ photons with frequency $\omega$ is given by

$$
\begin{gathered}
I_{\alpha}=\frac{e}{h} \int_{-\infty}^{\infty} \sum_{\beta=L B, R T}\left[1-f_{\alpha}(E)\right] f_{\beta}(E-\hbar \omega) T_{\alpha, \beta}^{-}(E) \\
-f_{\alpha}(E)\left[1-f_{\beta}(E+\hbar \omega)\right] T_{\alpha, \beta}^{+}(E) d E \\
T_{\alpha, \beta}^{-}(E)=N \operatorname{Tr}\left\{\Gamma_{\alpha}(E) G(E) M A_{\beta}(E-\hbar \omega) M^{\dagger} G^{\dagger}(E)\right\} \\
T_{\alpha, \beta}^{+}(E)=N \operatorname{Tr}\left\{\Gamma_{\alpha}(E) G(E) M^{\dagger} A_{\beta}(E+\hbar \omega) M G^{\dagger}(E)\right\}
\end{gathered}
$$

where $f_{\alpha}$ is the Fermi Dirac distribution function of lead $\alpha, A_{\alpha}=G \Gamma_{\alpha} G^{\dagger}$ is the spectral function of lead $\alpha, G$ and $G^{\dagger}$ are the retarded and advanced Green's functions and $M$ the electron-photon coupling matrix given by

$$
M_{m l}=\frac{e}{m_{0}}\left(\frac{\hbar \sqrt{\tilde{\mu}_{r} \tilde{\epsilon}_{r}}}{2 N \omega \tilde{\epsilon} c} F\right)^{\frac{1}{2}} \mathbf{e} \cdot \mathbf{p}_{m l}
$$

where $\tilde{\epsilon}, \tilde{\epsilon}_{r}$ and $\tilde{\mu}_{r}$ are the isotropic, homogenous permittivity, relative permittivity and relative permeability respectively, $F$ is the photon flux and $\mathbf{p}$ is the momentum operator. We take $\tilde{\mu}_{r}=1$. For the dielectric constant of MoSSe we have taken the average in-plane value of the $\mathrm{MoS}_{2}$ and $\mathrm{MoSe}_{2}$ parent TMD's. ${ }^{36}$

\section{Associated content}

Supporting Information. Details on screening from metallic leads, the projection of the lead coupling matrices and the length dependence of the current.

\section{Acknowledgement}

This work is partly funded by the Innovation Fund Denmark (IFD) under File No. 501600102, and the Lundbeck foundation (No. R95-A10510). The Center for Nanostructured 
Graphene (CNG) is sponsored by the Danish Research Foundation, Project DNRF103.

\section{References}

(1) Lu, A.-Y. et al. Nature Nanotechnology 2017, 12, 744-749.

(2) Zhang, J.; Jia, S.; Kholmanov, I.; Dong, L.; Er, D.; Chen, W.; Guo, H.; Jin, Z.; Shenoy, V. B.; Shi, L.; Lou, J. ACS Nano 2017, 11, 8192-8198.

(3) Qiu, D. Y.; da Jornada, F. H.; Louie, S. G. Nano Lett. 2017, 17, 4706-4712.

(4) Thygesen, K. S. 2D Mater. 2017, 4, 022004.

(5) Gunst, T.; Kaasbjerg, K.; Brandbyge, M. Phys. Rev. Lett. 2017, 118, 046601.

(6) Rivera, P.; Yu, H.; Seyler, K. L.; Wilson, N. P.; Yao, W.; Xu, X. Nature Nanotechnology $2018,1$.

(7) Kunstmann, J.; Mooshammer, F.; Nagler, P.; Chaves, A.; Stein, F.; Paradiso, N.; Plechinger, G.; Strunk, C.; Schüller, C.; Seifert, G.; Reichman, D. R.; Korn, T. Nature Physics 2018, 14, 801-805.

(8) Gong, Y.; Yuan, H.; Wu, C.-L.; Tang, P.; Yang, S.-Z.; Yang, A.; Li, G.; Liu, B.; Groep, J. v. d.; Brongersma, M. L.; Chisholm, M. F.; Zhang, S.-C.; Zhou, W.; Cui, Y. Nature Nanotechnology 2018, 13, 294-299.

(9) Chen, J.-W. et al. Nature Communications 2018, 9, 3143.

(10) Rasmussen, F. A.; Thygesen, K. S. J. Phys. Chem. C 2015, 119, 13169-13183.

(11) Cheng, Y. C.; Zhu, Z. Y.; Tahir, M.; Schwingenschlögl, U. EPL 2013, 102, 57001.

(12) Ji, Y.; Yang, M.; Lin, H.; Hou, T.; Wang, L.; Li, Y.; Lee, S.-T. The Journal of Physical Chemistry C 2018, 122, 3123-3129. 
(13) Ma, X.; Wu, X.; Wang, H.; Wang, Y. J. Mater. Chem. A 2018, 6, 2295-2301.

(14) Guan, Z.; Ni, S.; Hu, S. The Journal of Physical Chemistry C 2018,

(15) Wen, Y.-N.; Xia, M.-G.; Zhang, S.-L. Computational Materials Science 2018, 152, $20-27$.

(16) That the band gap almost exactly matches the optical band gap is fortuitous and a consequence of the cancelation between the missing self-energy effects in the band structure and excitonic effects in the 2D semiconductors.

(17) Dong, L.; Lou, J.; Shenoy, V. B. ACS Nano 2017, 11, 8242-8248.

(18) Smidstrup, S.; Stradi, D.; Wellendorff, J.; Khomyakov, P. A.; Vej-Hansen, U. G.; Lee, M.-E.; Ghosh, T.; Jónsson, E.; Jónsson, H.; Stokbro, K. Phys. Rev. B 2017, $96,195309$.

(19) Gunst, T.; Lü, J.-T.; Hedegård, P.; Brandbyge, M. Phys. Rev. B 2013, 88, 161401.

(20) Papior, N.; Gunst, T.; Stradi, D.; Brandbyge, M. Phys. Chem. Chem. Phys. 2015, 18, 1025-1031.

(21) Stradi, D.; Papior, N. R.; Hansen, O.; Brandbyge, M. Nano Lett. 2017, 17, 2660-2666.

(22) Wehling, T. O.; Novoselov, K. S.; Morozov, S. V.; Vdovin, E. E.; Katsnelson, M. I.; Geim, A. K.; Lichtenstein, A. I. Nano Lett. 2008, 8, 173-177.

(23) Wang, L.; Meric, I.; Huang, P. Y.; Gao, Q.; Gao, Y.; Tran, H.; Taniguchi, T.; Watanabe, K.; Campos, L. M.; Muller, D. A.; Guo, J.; Kim, P.; Hone, J.; Shepard, K. L.; Dean, C. R. Science 2013, 342, 614-617.

(24) Das, A.; Pisana, S.; Chakraborty, B.; Piscanec, S.; Saha, S. K.; Waghmare, U. V.; Novoselov, K. S.; Krishnamurthy, H. R.; Geim, A. K.; Ferrari, A. C.; Sood, A. K. Nature Nanotechnology 2008, 3, 210-215. 
(25) Efetov, D. K.; Kim, P. Phys. Rev. Lett. 2010, 105, 256805.

(26) Grover, S.; Joshi, A.; Tulapurkar, A.; Deshmukh, M. M. Scientific Reports 2017, 7, 3336.

(27) The current was scaled by the electrode cross-sectional area (width times the effective layer thickness $h \approx 7.5 \AA)$.

(28) Atomistix Toolkit version 2018.06, Synopsys QuantumWise A/S.

(29) ASTM, Astm 2013, 03, 1-21.

(30) Palsgaard, M.; Markussen, T.; Gunst, T.; Brandbyge, M.; Stokbro, K. Phys. Rev. Applied 2018, 10, 014026.

(31) For the silicon device we have included phonon-assisted processes ${ }^{37}$ that generates most of the photo-current due to the indirect band gap. For MoSSe we did not include phonon coupling and the direct transition already gives a significant current. The estimate here then gives a lower limit to the photo-current generated without phonon-assisted processes in MoSSe.

(32) Brandbyge, M.; Mozos, J.-L.; Ordejón, P.; Taylor, J.; Stokbro, K. Phys. Rev. B 2002, $65,165401$.

(33) Henrickson, L. E. J. Appl. Phys. 2002, 91, 6273-6281.

(34) Zhang, L.; Gong, K.; Chen, J.; Liu, L.; Zhu, Y.; Xiao, D.; Guo, H. Phys. Rev. B 2014, 90, 195428.

(35) Chen, J.; Hu, Y.; Guo, H. Phys. Rev. B 2012, 85, 155441.

(36) Laturia, A.; Put, M. L. V. d.; Vandenberghe, W. G. npj 2D Materials and Applications 2018, 2, 6 . 
(37) Gunst, T.; Markussen, T.; Palsgaard, M. L. N.; Stokbro, K.; Brandbyge, M. Phys. Rev. B 2017, 96, 161404. 


\section{Graphical TOC Entry}

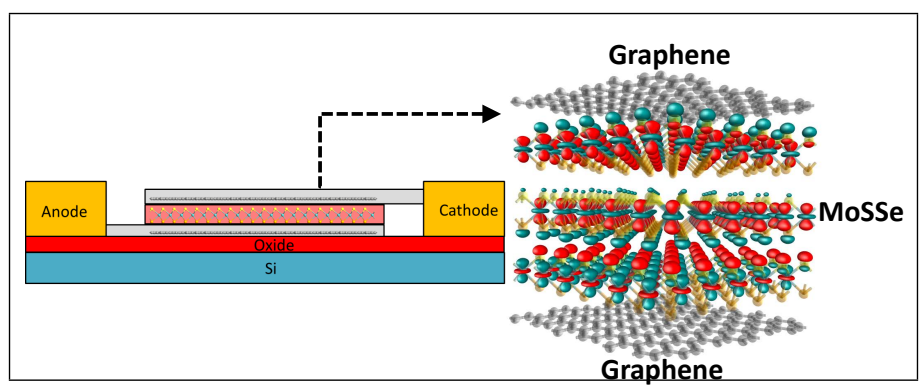

